\title{
ESOPHAGEAL STRICTURE ASSESSMENT BY ESOPHAGOGRAM: A PICTORIAL REVIEW OF DIFFERENTIAL DIAGNOSIS
}

\author{
Marcio Aloisio Bezerra Cavalcanti Rockenbach¹, \\ Ivan Morzoletto Pedrollo², Antônio Carlos Maciel ${ }^{1}$
}

\begin{abstract}
The purpose of this article is to review the most frequent conditions associated with esophageal strictures on esophagogram. The most common causes include caustic ingestion, radiation therapy, malignancy, extrinsic compression, gastroesophageal reflux disease, infectious esophagitis, systemic diseases and membranes and rings. Adequate characterization of the stricture is necessary for formulation of the best diagnostic hypothesis and can be used to distinguish between malignant and non-malignant conditions.
\end{abstract}

Keywords: Esophageal diseases; esophageal stenosis; esophagus; esophageal neoplasms; esophageal achalasia; radiography; radiology; radiotherapy

One of the most common symptoms in clinical practice is dysphagia. Although many new examination techniques have arisen, esophagogram is still a valuable tool in the initial assessment of these patients. The purpose of this article is to review the most frequent conditions associated with esophageal strictures. Those are common diseases which must be mastered by all radiologists.

\section{Caustic Ingestion}

Ingestion of a caustic agent (acid or alkaline), whether accidental or intentional, causes extensive esophagitis due to ulceration and necrosis of the mucosa ${ }^{1}$. After about three months, due to the process of healing and fibrosis, there is stenosis formation, usually extensive, which may be localized (usually at the level of the aorta and the left main bronchus) or diffuse (Figure 1). The esophagogram is used to assess the extent of stenosis area and to diagnose complications, such as fistula. There is an increased risk of squamous cell carcinoma of the esophagus, which can manifest as a new area of irregularity or nodularity in the affected area.

It is worth mentioning that it is preferred to use water-soluble contrast agents instead of barium in the acute stage or when a complication is suspected, such as fistula, as this type of contrast is less irritant. For chronic cases, barium is the usually chosen contrast because it provides greater radiographic details than water-soluble agents ${ }^{2}$.

\section{Radiation Therapy}

Damage to the esophagus may be caused when a radiation dose above $50 \mathrm{~Gy}$ is used $^{3}$, causing acute esophagitis. Although most cases are self-limited, some patients may develop stenosis in 4-8 months after completion of radiotherapy, leading to dysphagia. The main finding is a concentric smooth stenosis area in a previously irradiated location ${ }^{1}$ (Figure 2).

\section{Malignancy}

There are two main histological types of esophageal cancer: squamous cell carcinoma and adenocarcinoma. Squamous cell carcinoma is responsible for most cases (about 50-70\%). It usually affects the proximal and middle thirds
Clin Biomed Res. 2018;38(4):377-383

1 Serviço de Radiologia, Hospital de Clínicas de Porto Alegre (HCPA). Porto Alegre, RS, Brasil.

2 Núcleo de Radiologia do Abdômen, Serviço de Radiologia, Hospital Moinhos de Vento (HMV). Porto Alegre, RS, Brasil.

Corresponding author: Marcio Aloisio Bezerra Cavalcanti Rockenbach marcioabcr@gmail.com Serviço de Radiologia, Hospital de Clínicas de Porto Alegre (HCPA) Rua Ramiro Barcelos, 2350. 90035-903, Porto Alegre, RS, Brasil. 


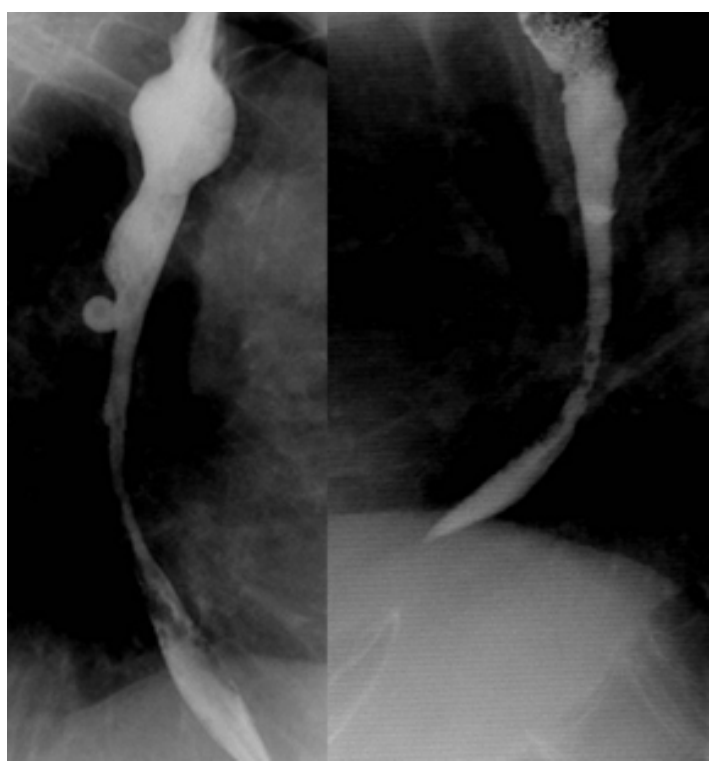

Figure 1: Caustic ingestion. Female patient, 72 years. Extensive area of stenosis and parietal irregularity affecting the distal two-thirds of the thoracic esophagus, with esophageal dilatation and upstream stasis of contrast medium. Also, a diverticular formation is observed, located in the left posterolateral wall of the middle third of the thoracic esophagus (mid-esophageal diverticulum).

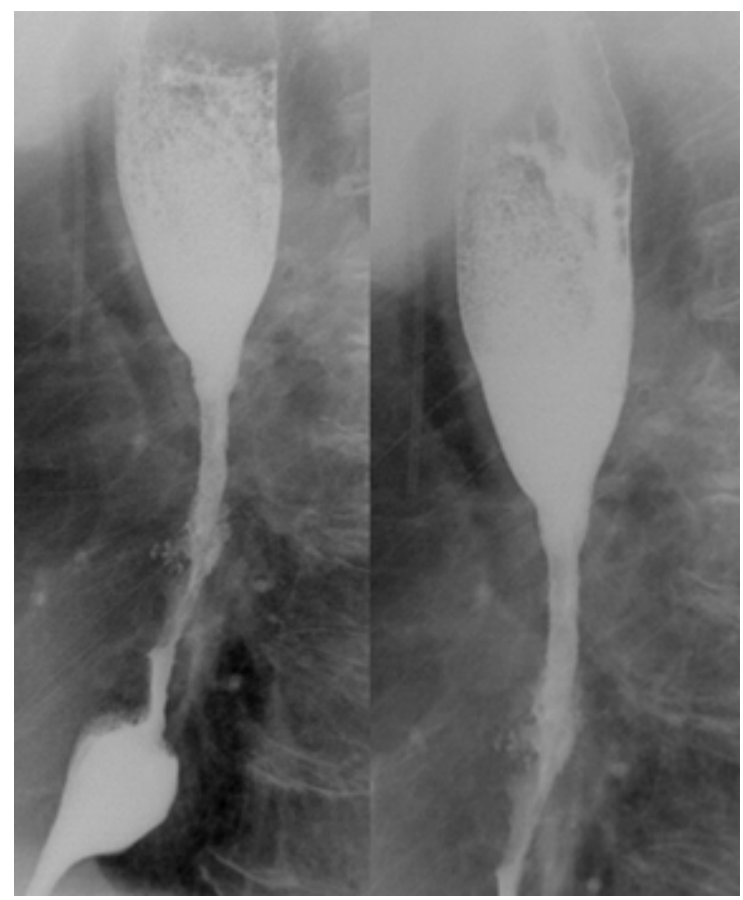

Figure 2: Follow-up study after esophageal cancer radiotherapy. Female patient, 78 years. Parietal irregularities and esophageal stenosis with possible areas of ulceration, probably due to radiotherapy. Distinguishing it from neoplastic recurrence is challenging because of similarities in the findings. Endoscopic study or computed tomography scan may help in the differential diagnosis. of the esophagus (Figures 3 and 4), and its main risk factors are smoking and alcoholism ${ }^{4}$. Adenocarcinoma, which accounts for about $30-50 \%$ of cases, is usually associated with gastroesophageal reflux disease (GERD) and Barrett's esophagus, usually affecting the lower third of the esophagus (Figure 5).

Because the esophagus does not have a serous layer, tumor spread is facilitated. Therefore, diagnosis is often delayed. Symptoms usually arise from compressive effects and include dysphagia, epigastric pain, cough, weight loss and hoarseness.

Esophagography has a high sensitivity for diagnosis of malignancy ${ }^{5}$. Tumor findings in early stages include plates (often with central ulcers), sessile polyps and focal irregularities in the esophageal wall. In more advanced stages, they may appear as infiltrating, polypoid, ulcerate or even varicoid lesions. Infiltrative lesions are mostly associated with narrowing of the esophageal lumen. Other findings include nodularity or ulceration of the mucosa and abrupt transition between the area of the lesion and the normal esophagus.

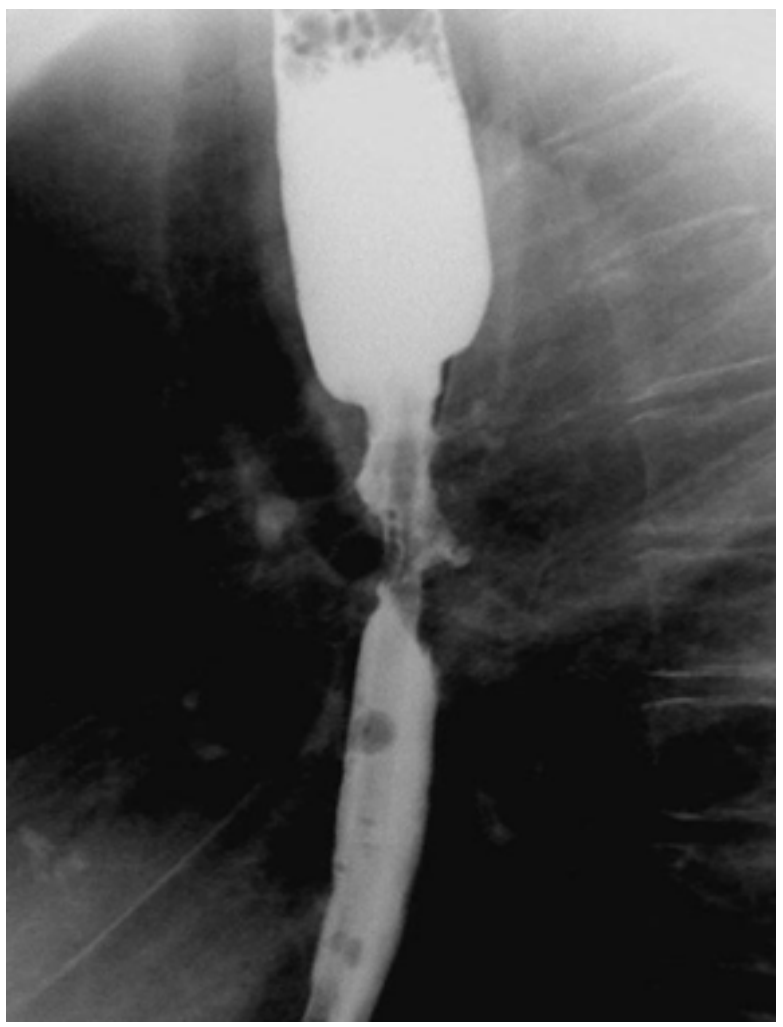

Figure 3: Esophageal squamous cell carcinoma. Female patient, 60 years. Concentric stenosis area in the middle third of the esophagus, about $4.3 \mathrm{~cm}$ long, with irregular contours and sharp boundaries, determining stasis of contrast medium and upstream dilation of the esophagus. The dilated esophagus causes compression and anterior displacement of the trachea. 


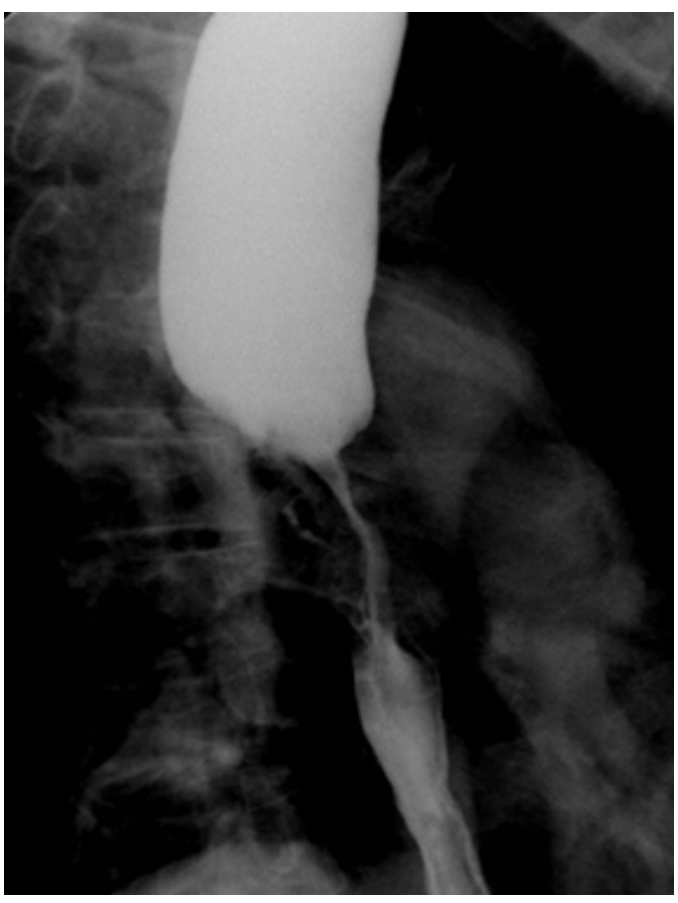

Figure 4: Esophageal squamous cell carcinoma. Male patient, 71 years. Irregular concentric stenosis area in the middle third of the thoracic esophagus, measuring about $7.8 \mathrm{~cm}$ long, with upstream dilation of the esophagus and contrast medium stasis.

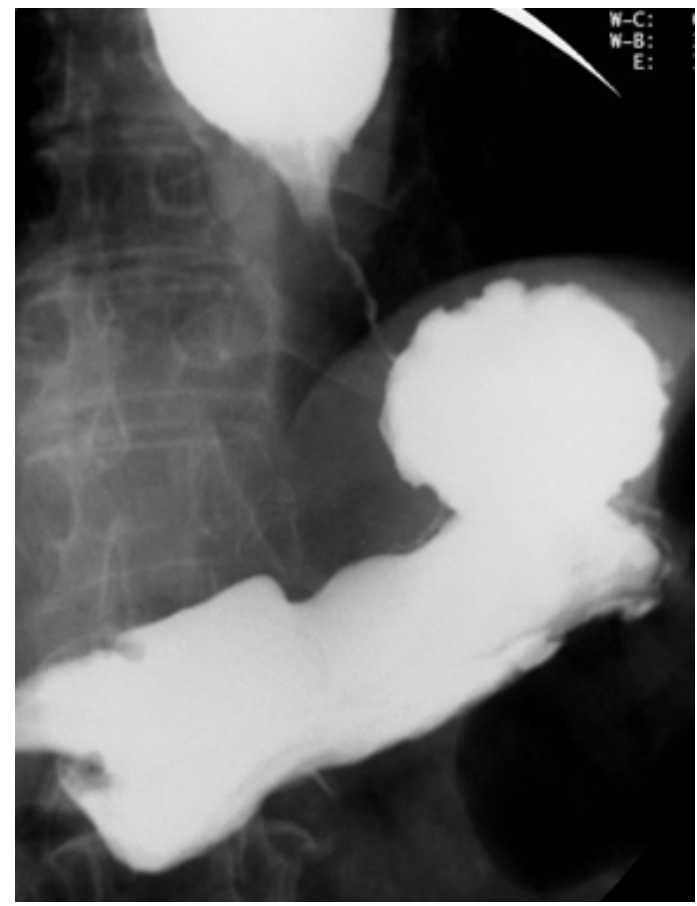

Figure 5: Esophageal adenocarcinoma. Male patient, 85 years. Concentric stenosis of the distal esophagus, with irregular borders, with dilation of the esophagus and contrast medium stasis. Distinguishing it from achalasia may be difficult in many cases.
Other less common malignant lesions of the esophagus that may eventually manifest as stenosis include lymphoma (Hodgkin's and non-Hodgkin's), carcinosarcoma, leiomyosarcoma, melanoma and Kaposi's sarcoma ${ }^{1}$.

\section{Extrinsic Compression}

Esophageal strictures may also be determined by mass effect of thoracic structures ${ }^{6}$ :

- Thyroid: increased thyroid may cause dislocation of the cervical esophagus or, in cases of massive goiter, of the upper thoracic esophagus (Figure 6);

- Aberrant right subclavian artery (lusory artery): the subclavian artery arises directly as the fourth branch of the aortic arch. In $80 \%$ of cases, its course is posterior to the esophagus, causing an impression on its posterior wall;

- Structures of the spine (Figure 7): prominent osteophytes may cause displacement or narrowing of the esophageal lumen, particularly in C5-C6 (where the esophagus is attached to the cricoid cartilage) or in the upper thoracic esophagus;

- Malignancy: stenosis may also result from direct invasion of an adjacent lung cancer or from compression by a mediastinal neoplasm.

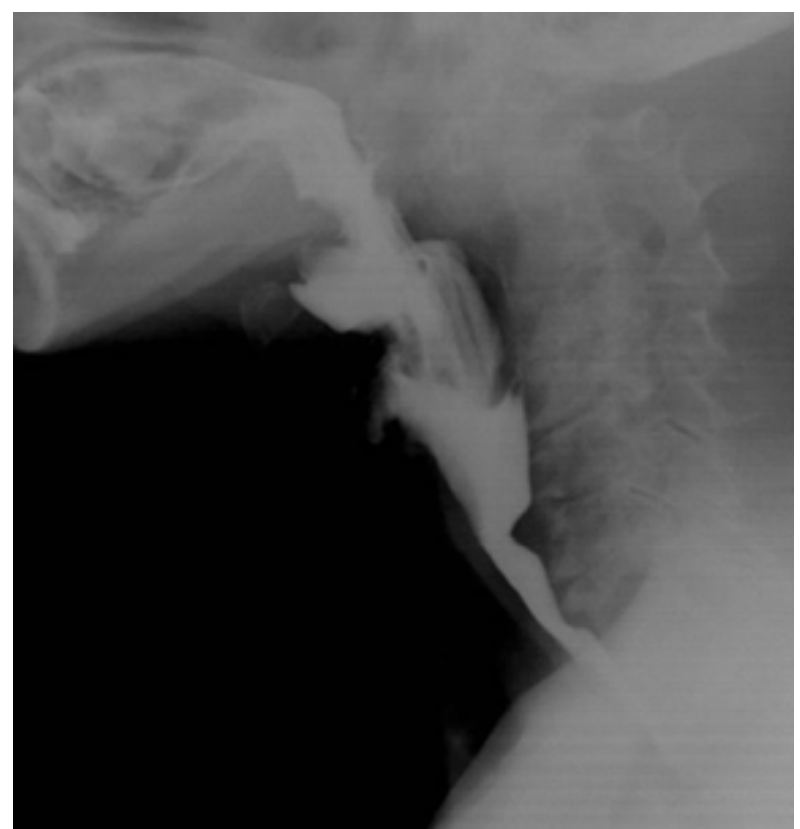

Figure 6: Extrinsic compression by goiter. Female patient, 49 years. Reduction in the diameter and lateral displacement of the cervical esophagus by increased thyroid volume. 


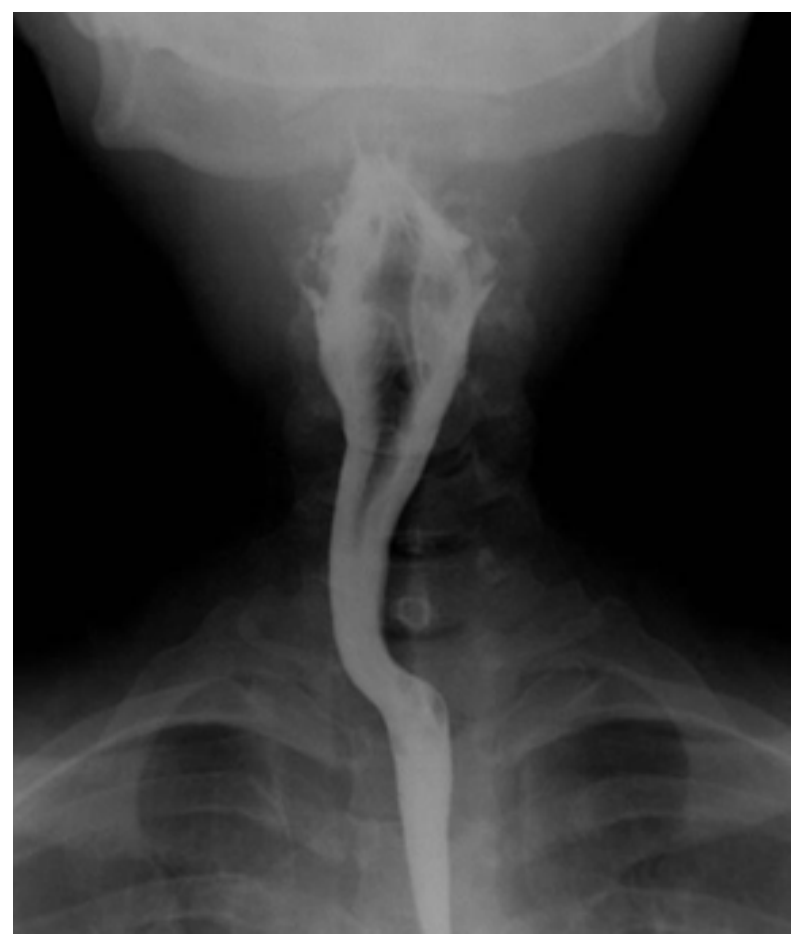

Figure 7: Extrinsic compression caused by the spine. Male patient, 103 years. Somatic anterior cervical osteophytes causing an impression on the posterior wall of the esophagus at the C5-C6 level, due to disc degeneration. Also, incomplete cricopharyngeal muscle relaxation is seen.

\section{Gastroesophageal Reflux Disease (GERD)}

Because of its high incidence in the general population, GERD is the leading cause of esophageal strictures $^{5}$. The characteristic initial findings of esophagitis, best evaluated on double-contrast studies, demonstrate a fine granular or nodular pattern of the distal esophageal mucosa, followed by small erosions or ulcerations ${ }^{4}$. The stenotic manifestations due to GERD result from an advanced stage of the disease, caused by scarring processes, which demonstrate conical and concentric tapering (sometimes asymmetric) of the distal esophagus, 2-3 $\mathrm{cm}$ above a hiatal hernia (Figure 8).

\section{Infectious Esophagitis}

The main causes include Candida albicans (Figure 9), herpes simplex virus, cytomegalovirus infections (Figure 10) and HIV esophagitis ${ }^{5}$. Esophagography plays an important role in the diagnosis of esophageal candidiasis, as characteristic findings indicate treatment without the need for biopsy. Typical findings are pseudomembranes and coalescing plates where the barium lodges, which determine a grossly irregular pattern of the walls. Herpetic esophagitis is characterized by the presence

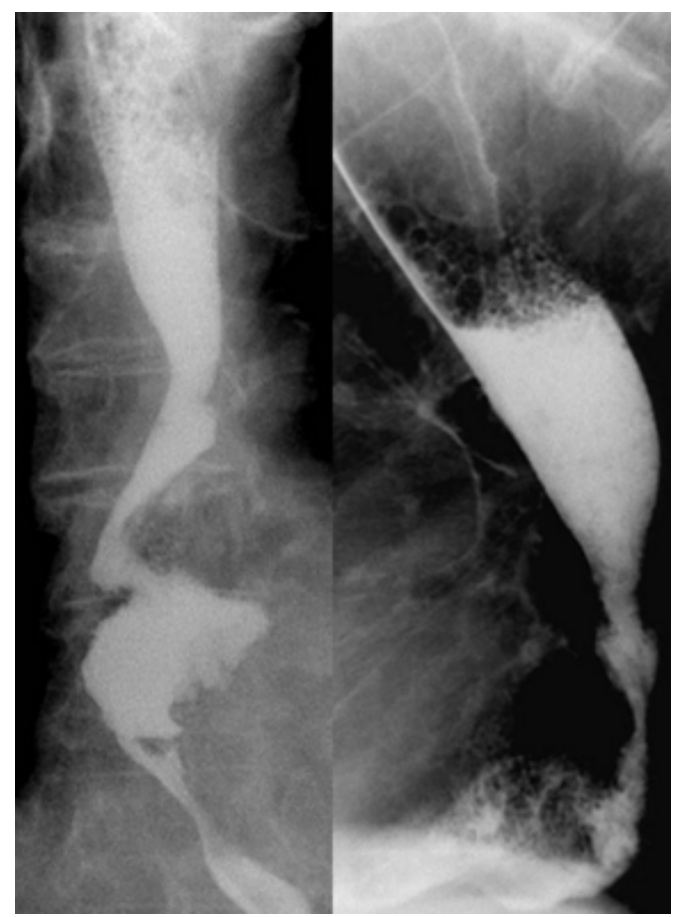

Figure 8: Chronic gastroesophageal reflux disease with previous dilation - follow-up study Female patient, 97 years. Progressive concentric narrowing of the distal esophagus at the level of the gastroesophageal junction, associated with esophageal shortening. Also, a sliding non-reducible hiatal hernia and small nodular images in the distal esophagus are seen, which may correspond to small ulcerations.

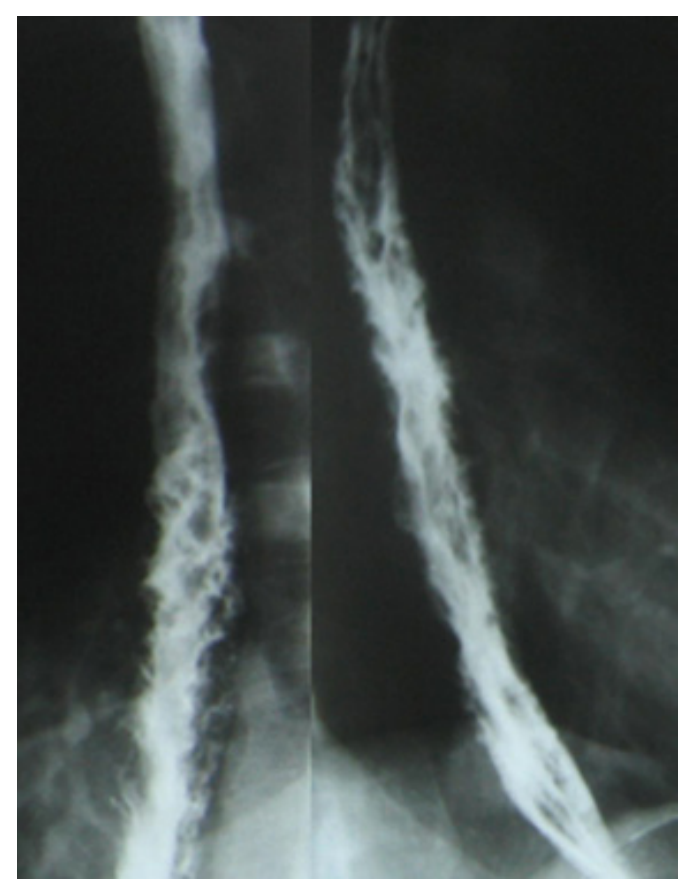

Figure 9: Candida albicans esophagitis. Grossly irregular characteristic pattern (shaggy pattern), determined by multiple ulcerations and pseudomembranes. 


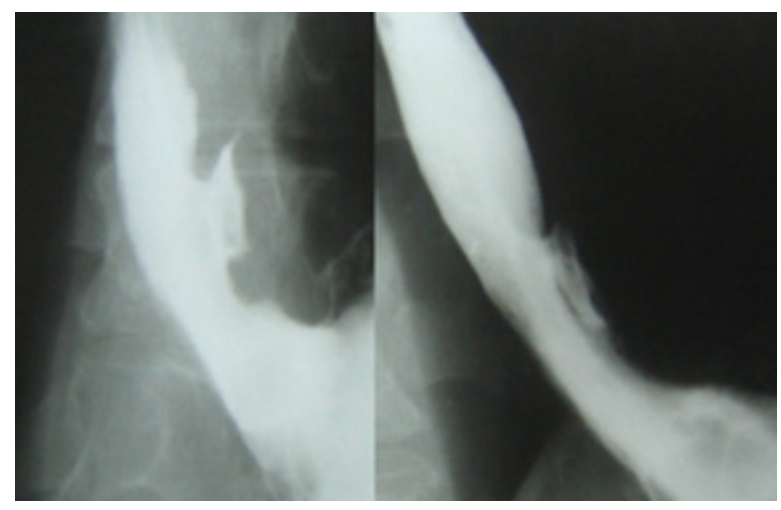

Figure 10: Cytomegalovirus esophagitis. Large isolated parietal ulceration surrounded by a radiolucent halo, related to edema.

of small ulcers distributed between segments of the normal mucosa, without plaques ${ }^{4}$. Cytomegalovirus esophagitis, frequently associated with HIV, manifests most commonly as large ulcers, sometimes isolated, flat and surrounded by a radiolucent halo related to mucosal edema. HIV-related esophagitis has similar characteristics to cytomegalovirus esophagitis, making it difficult to distinguish those entities based only on esophagogram ${ }^{1}$.

\section{Systemic Diseases}

Many systemic diseases can affect the esophagus and cause strictures. Achalasia is an esophageal motility disorder characterized by absent primary peristalsis and incomplete relaxation of the lower esophageal sphincter which can be secondary to systemic diseases such as Chagas disease (Figure 11) and Allgrove syndrome ${ }^{7}$ (Figure 12). Chagas disease is a common cause of achalasia in tropical regions. Collagen diseases such as scleroderma and systemic lupus erythematosus may cause esophageal dysmotility and stenosis. Other less common skin diseases that affect mucous membranes, such as dystrophic epidermolysis bullosa (Figure 13) or dyskeratosis congenita (Figure 14), may also affect multiple organs, including the esophagus ${ }^{5}$.

\section{Membranes and Rings}

The esophageal membranes consist of mucosal folds located at the cervical esophagus or hypopharynx and they present with shelf-like filling defects with 1-2 mm height ${ }^{5}$. They are predominantly located anteriorly but may be circumferential, causing obstruction and upstream dilatation (Figure 15). They may also be single or multiple and associated with other conditions (pemphigus, epidermolysis bullosa dystrophica, GERD).

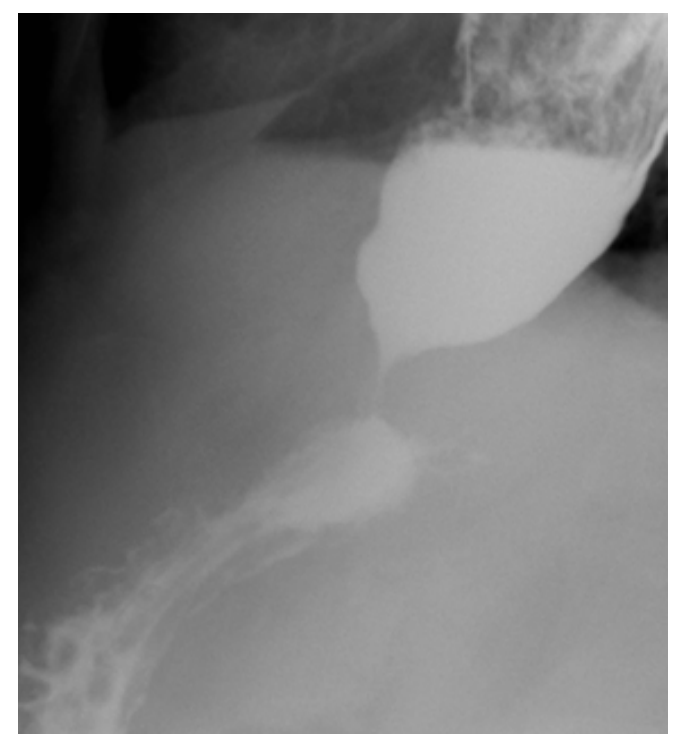

Figure 11: Achalasia secondary to Chagas disease. Male patient, 59 years. Narrowing of the distal esophagus, causing upstream dilatation and contrast medium stasis.

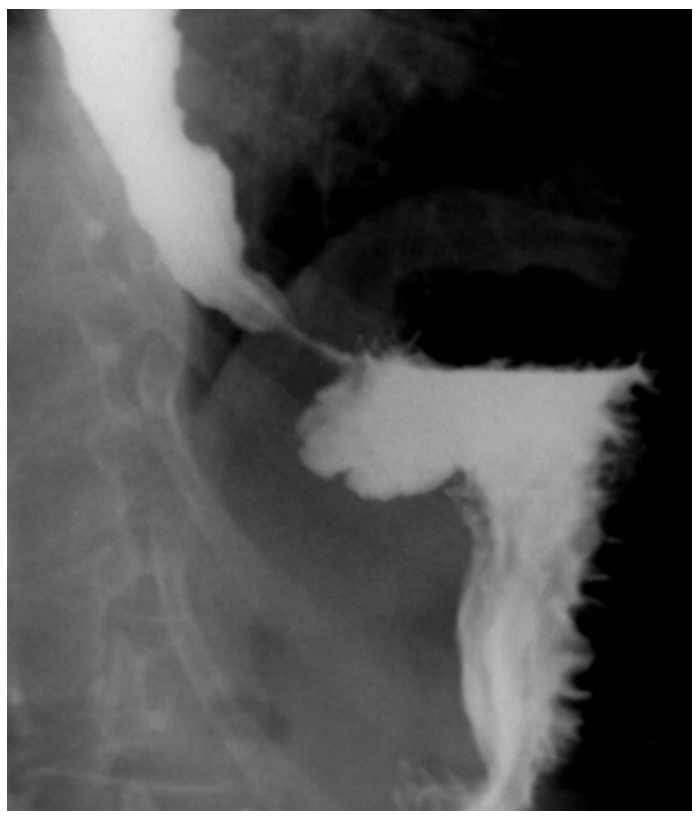

Figure 12: Allgrove syndrome. Female patient, 40 years. Achalasia associated with Allgrove syndrome, characterized by adrenal insufficiency, achalasia of the cardia, alacrima and autonomic abnormalities (4A syndrome).

Esophageal rings are concentric and flat extensions of normal esophageal tissue (which may contain mucosa, submucosa and muscle), with thickness generally ranging from 3-5 $\mathrm{mm}$. Most are asymptomatic and consist of an incidental finding. The Schatzki ring is the most common example, manifesting as a 


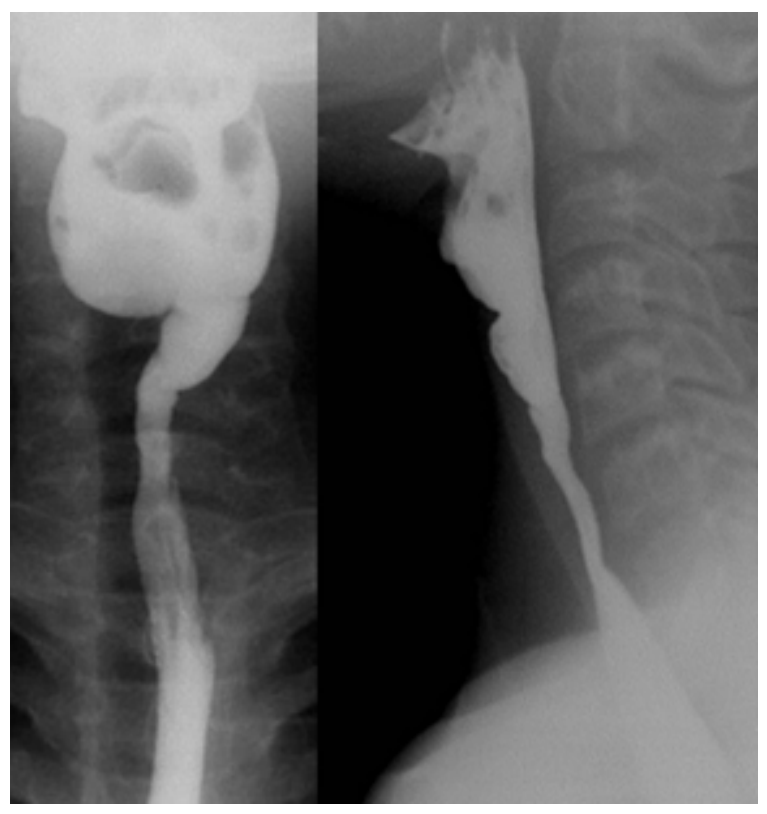

Figure 13: Epidermolysis bullosa. Female patient, 3 years. Smooth segment stenosis in the proximal third of the thoracic esophagus.

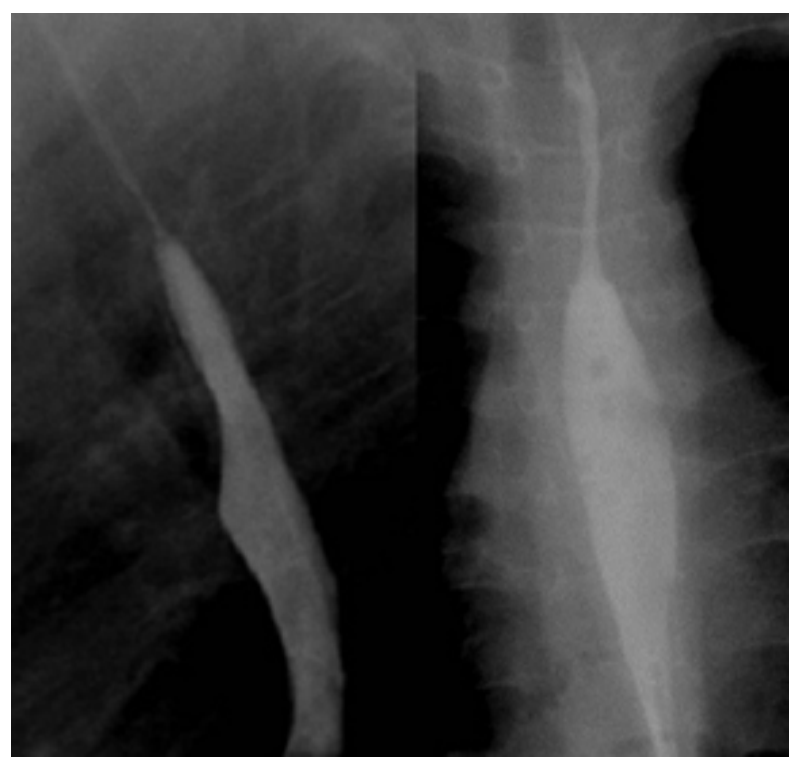

Figure 14: Dyskeratosis congenita. Male patient, 13 years. Important concentric stenosis area (determined by membranes), with regular contours, situated on pharyngoesophageal transition, determining upstream dilatation and contrast medium stasis.

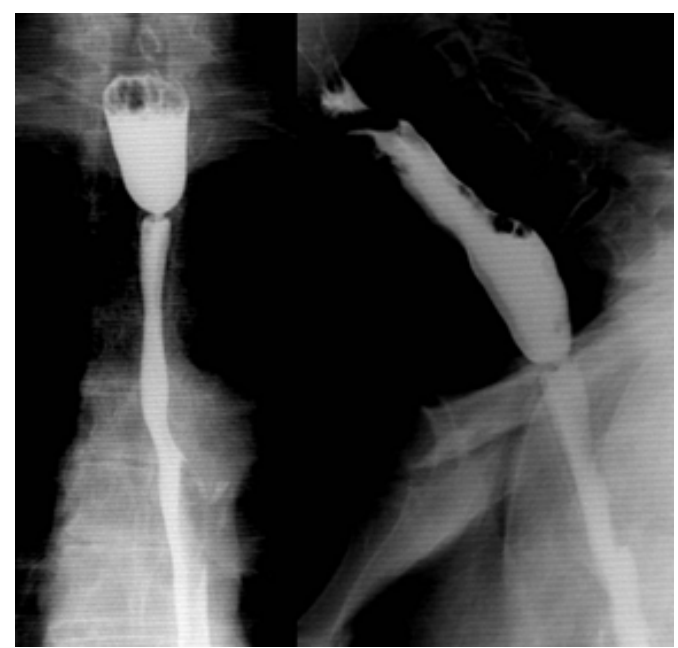

Figure 15: Esophageal membrane. Male patient, 78 years. Annular stenosis of the cervicothoracic esophageal junction.

flat concentric ring on the distal esophagus, typically associated with a hiatal hernia ${ }^{8}$. Dietary treatment is usually sufficient, but, in refractory cases, dilatation may be required.

\section{CONCLUSION}

The esophagography is a test that provides important information on diseases of the gastrointestinal tract. In comparison with other evaluation methods, the main advantages of the esophagogram are low costs and a dynamic evaluation of the esophageal function. However, other diagnostic methods might be needed to confirm the suspected diagnosis or to further evaluate the extent of the disease, such as computed tomography and endoscopy (with biopsy, when needed). Because it still is a valuable tool in esophageal assessment, all radiologists must be trained to properly interpret esophagogram findings.

Esophageal strictures are one of the most common findings and are related to a wide variety of diseases, whether benign or malignant. Proper characterization of imaging findings and correlation with clinical history are essential for proper interpretation and for diagnostic hypothesis construction.

\section{Conflicts of Interest}

The authors declare no conflicts of interest.

\section{REFERENCES}

1. Levine MS, Rubesin SE. Diseases of the esophagus: diagnosis with esophagography. Radiology.
2005;237(2):414-27. http://dx.doi. org/10.1148/radiol.2372050199. PMid:16170017.
2. Contini $\mathrm{S}$, Scarpignato C. Caustic injury of the upper gastrointestinal tract: a comprehensive review. World 
J Gastroenterol. 2013;19(25):391830. http://dx.doi.org/10.3748/wjg.v19. i25.3918. PMid:23840136.

3. Karasick S, Lev-Toaff S. Esophageal strictures: findings on barium radiographs. AJR Am J Roentgenol. 1995;165(3):561-5. http://dx.doi. org/10.2214/ajr.165.3.7645471. PMid:7645471.

4. Kumbasar B. Carcinoma of esophagus: radiologic diagnosis and staging. Eur J Radiol. 2002;42(3):17080. http://dx.doi.org/10.1016/S0720048X(02)00030-X. PMid:12044696.
5. Levine MS, Rubesin SE, Laufer I. Barium esophagography: a study for all seasons. Clin Gastroenterol Hepatol. 2008;6(1):11-25. http:// dx.doi.org/10.1016/j.cgh.2007.10.029. PMid:18083069.

6. Carucci LR, Turner MA. Dysphagia revisited: common and unusual causes. Radiographics. 2015;35(1):105-22. http://dx.doi. org/10.1148/rg.351130150. PMid:25590391.
7. Woodfield CA, Levine MS, Rubesin SE, Langlotz CP, Laufer I. Diagnosis of primary versus secondary achalasia: reassessment of clinical and radiographic criteria. $A m \mathrm{~J}$ Roentgenol. 2000;175(3):72731. http://dx.doi.org/10.2214/ ajr.175.3.1750727. PMid:10954457.

8. Baker ME, Einstein DM. Barium esophagram: does it have a role in gastroesophageal reflux disease? Gastroenterol Clin North Am. 2014;43(1):47-68. http://dx.doi. org/10.1016/j.gtc.2013.11.008. PMid:24503359.

Received: Mar 26, 2018 Accepted: Oct 4, 2018 